\title{
Approach to Dyslipidemia, Lipodystrophy, and Cardiovascular Risk in Patients with HIV Infection
}

\author{
J. Gregory Troll
}

Published online: 23 December 2010

(C) The Author(s) 2010. This article is published with open access at Springerlink.com

\begin{abstract}
There is a significant prevalence $(20 \%-80 \%$ depending on the population and the study) of lipid disorders and other cardiovascular risk factors in people living with HIV infection. This review focuses on HIV and HIV treatment-associated metabolic and cardiovascular concerns, including dyslipidemias, lipodystrophy syndromes, endothelial dysfunctions, and associated metabolic events such as insulin resistance. Emerging hypotheses of the underlying pathophysiology of these issues, with impact on selection of specific antiretroviral treatment (ART) strategies, therapy, and preventive approaches to decreasing cardiovascular risk and other problems associated with these syndromes are discussed. Screening for cardiovascular risk as part of the decision of starting antiretroviral therapy, and during care of patients with HIV regardless of ART therapy status, is suggested with particular areas of focus. Statins, other hyperlipidemic therapies, treatment for specific problems arising due to lipodystrophy, and implications on ART selection to avoid drug interactions and adverse effects are also discussed.
\end{abstract}

Keywords HIV - Lipodystrophy · Dyslipidemia .

Statin therapy $\cdot$ Cardiovascular risk prevention $\cdot$ Review

\section{Introduction}

In the 1980s, as the first phase of the AIDS epidemic was raging, many of us were reeling from the impact of trying

J. G. Troll $(\bowtie)$

Touro University College of Osteopathic Medicine,

1310 Club Drive,

Vallejo, CA 94592, USA

e-mail: greg.troll@tu.edu to help patients desperately ill and dying around us. We commiserated that, if we could not find a cure, it would be a great step forward if we could just be able to make AIDS a manageable condition, such as diabetes or hypertension. A few years later, but before the breakthrough that was highly active antiretroviral therapy (HAART), a colleague mentioned to me, with a hint of both celebration and irony, that he was working with an HIV patient on issues of hyperlipidemia and atherosclerosis. The subtext was that our HIV patients rarely had lived long enough to develop those issues, and that perhaps we were finally getting somewhere with the limited advances we had made up to that point. In 1995, with the emergence of HAART, reports began fairly early on of "crix belly" - emerging signs of lipodystrophy assumed early on to be related to protease inhibitors. The prevalence of these syndromes in the HIVpositive population is somewhere between $20 \%$ and $80 \%$, depending on the study and population referred to. Apparently, we should have been more careful in what we had asked for, as we had gotten, eerily, precisely that. In the industrialized world, at least, HIV was now (with profound thanks) a chronic manageable condition, but not merely like diabetes and cardiovascular disease, as it turned out, including those conditions. It may be, in reality, that a great deal of these cardiovascular complications emerged not simply as a complication of therapy, but due to increased survival allowing chronic effects of HIV infection, other than immunodeficiency, to exert significant pathologic effects. Supporting this possibility are the findings that HIV viremia and/or immunodeficiency has been shown to be associated with endothelial dysfunction [1], hypercoagulability $[2,3]$, vascular damage $[4 \bullet, 5 \bullet]$, and inflammation with C-reactive protein (CRP) elevation [6]. In these instances, these effects are independent of antiretroviral therapy (ART). Nonetheless, there is also a 
great deal of evidence implicating ART, and certain agents in particular appear to play a causative role in the lipodystrophy syndromes.

This review focuses on HIV and HIV treatmentassociated metabolic and cardiovascular concerns. Overall, HIV and HIV therapy-associated vascular effects include dyslipidemias, lipodystrophy syndromes, and endothelial and other dysfunctions. Metabolic effects, such as insulin resistance, are also important to address as part of the complex of lipodystrophy and cardiovascular risk in HIV patients and will be touched upon. Research on lipodystrophy and dyslipidemias has focused on population and clinical trials, research on adipocyte cell culture lines, and on overall hepatic function and markers of inflammation. Although the research on adipocytes reveals complex and interesting interactions with regulatory systems that may explain many features of metabolic syndromes, they tend to divert attention from the contribution of overall inflammation, macrophage turnover, hepatic steatosis, and other features of HIV infection and ART that contribute to the whole picture of lipodystrophy and dyslipidemias [7]. This article covers preventive and therapeutic implications, focusing especially on the use of statins and other modalities to treat these associated complications of HIV and its treatment modes.

\section{Overview of the Problem}

Patients living with HIV and taking ART are subject to developing lipodystrophy syndromes that include dyslipidemias (alterations of cholesterol and triglyceride profiles associated with increased risk of atherogenic progression), similar to metabolic syndrome X. Lipodystrophy syndromes also are associated with fat maldistribution, presenting frequently as abdominal visceral obesity and cervical fat pad accumulation (ie, buffalo hump), reminiscent of Cushing's syndrome (and sharing in common with Cushing's syndrome a role for cortisol-stimulated lipogenesis in visceral adipocytes). An additional feature of fat maldistribution is peripheral subcutaneous lipoatrophy, usually associated with mitochondrial dysfunction, but also possibly triggered by other pathways leading to adipocyte atrophy and apoptosis. Lipoatrophy had been seen prior to the advent of combination ART in conjunction with wasting syndrome in treatmentnaïve patients with HIV, but lipoatrophy as part of the bigger picture of fat maldistribution has been associated primarily with some of the nucleoside reverse transcriptase inhibitors, particularly the thymidine analogs $\mathrm{d} 4 \mathrm{t}$ (stavudine) and AZT (zidovudine). As an additional risk factor for cardiovascular disease, endothelial dysfunction (as reflected as changes in levels of von Willebrand factor, plasminogen activator inhibitor 1 [PAI-1], tissue plasminogen activator [t-PA], soluble adhesion molecules [sE-selectin], intercellular adhesion molecules [ICAM] and decreases in flow-mediated dilation) is present in HIV-infected people, both those who are receiving antiretroviral therapy and those who are treatment naïve [8]. These metabolic and vascular disturbances appear to pose increased cardiovascular risk in HIV patients just as they do in the general population [9*0]. Although lipodystrophy gained recognition and attention shortly after the advent of HAART, and seemed obviously connected with antiretroviral therapy, some population studies have failed to demonstrate this correlation. Some patients improve, in part, with ART, and it remains possible that these effects are of mixed origin, possibly related to both HIV infection itself and to its therapy [10].

Because these syndromes cannot always be avoided, even with careful selection of ARTs, there is a general sentiment that treatment for patients with these problems, as they appear in the HIV-positive population, is indicated to prevent cardiovascular morbidity and mortality, although the outcome data in this particular population are limited [11]. Preventive strategies include diet and lifestyle changes, antihyperlipidemic therapies, treatment focusing on associated insulin resistance and diabetes risk, and codiagnosis.

\section{Underlying Mechanisms of Treatment-Associated Lipodystrophy Syndromes}

There are several theoretical mechanisms by which ART contributes to lipodystrophy syndromes. Protease inhibitors impair adipocyte differentiation through multiple biochemical interactions with adipocyte proteosomal gene expression systems, down-regulation of cellular retinoic acid binding protein (CRABP), sterol regulatory binding protein levels with resultant dysregulation of gene expression stimulated by cortisol, activation of the adipocyte renin-angiotensin system and adipokine effects (including adiponectin and leptin among others), and decrease in peroxisomal proliferatoractivator receptors (PPAR) $\alpha$ and $\gamma$. A number of other potential biochemical mechanisms probably play a part in lipodystrophy syndromes, involving other sterol intermediaries, the interaction of oxidative stress, inflammation, mediated by tumor-necrosis factor- $\alpha$ (TNF- $\alpha$ secretion and receptor pathways, and several other pathways that may reflect host genetics and HIV effects on inflammatory states in addition to protease inhibitor pharmacologic side effects. These effects are generally manifested as adipose hypertrophy, especially in visceral adipose stores, increased triglycerides, decreased high-density lipoprotein (HDL), adipocyte insulin resistance, hypertension, and tendency to type 2 diabetes mellitus. In this way, these manifestations overlap with those seen in the general HIV-seronegative 
population and are described as metabolic syndrome (or metabolic syndrome $\mathrm{x}$ ). Although protease inhibitors are the pharmacologic agents most responsible for these effects in patients on HAART, there is evidence that nucleoside analogs also contribute. Nonetheless, switching patients to protease inhibitor-sparing regimens is often at least a partially successful strategy in mitigating these adipocyte effects. Changing antiretroviral therapy as dictated by optimal lipid effects is, however, not always feasible for adequate antiretroviral control. Juggling antiretroviral regimens to avoid untoward lipid effects also may require agents that are not always available or to which the patient's infection has developed resistance.

As mentioned above, thymidine analogue nucleoside reverse transcriptase inhibitors (tNRTIs) are a risk factor for mitochondrial dysfunction associated with both dyslipidemias and lipoatrophy (particularly in subcutaneous adipocytes) [12] Changing patients from thymidine analogs to NRTIs that don't have these effects has demonstrated beneficial effects on lipoatrophy [13]. Other therapeutic possibilities are mentioned below.

Patients with mitochondrial dysfunction manifesting as more systemic disease, including asymptomatic or symptomatic lactic acidosis, require more urgent therapy. The mortality rate from symptomatic lactic acidosis is high and this requires urgent intervention and hospitalization.

In addition to the above antiviral drug class effects, nonnucleoside reverse transcriptase inhibitors (NNRTIs), such as efavirenz, have been associated with in vitro altered deposition of triglycerides in the adipocyte [14], although the contribution to clinical effects from these findings has not been made clear as of yet.

\section{Treatment and Prevention}

As a rule, prevention should focus initially on diet and lifestyle and changing the ART. Adding antihyperlipidemic, antihypertensive, and insulin-sensitizing drugs to the regimen would normally be a subsequent step. As in HIVnegative patients, exercise and caloric restriction do have the potential to improve visceral obesity and reverse some of the changes in metabolic syndrome [15]. Visceral adiposity and dyslipidemia are both targets for which there is evidence for improvement with exercise and diet. There is less evidence for lipoatrophy responding to these measures. It is not clear to what degree the component of endothelial dysfunction improves with diet and exercise in the HIV-infected population. Depending on the severity and risk factors, it would seem reasonable to start patients with dyslipidemias and metabolic syndrome on diet and lifestyle interventions as a first step prior to starting them on more pharmacotherapy (which always carries with it the potential for drug-drug interactions.) In patients with lipoatrophy with or without other manifestations of mitochondrial toxicity, switching from d4T (stavudine) or AZT (zidovudine) to abacavir or tenofovir, or, alternatively, switching to a nucleoside-sparing regimen with several protease inhibitors, appears to improve gradually the clinical manifestations, with some eventual reversal of the subcutaneous atrophy $[16,17]$. The freedom of designing antiretroviral regimens to avoid complications like lipodystrophy and dyslipidemia, however, is constrained by the importance of adequate antiretroviral activity, often complicated by viral resistance, and at times the limited availability and cost of some antiretroviral choices over others. Stavudine, the nucleoside analog with the highest propensity for causing mitochondrial dysfunction, is relatively inexpensive and often has better availability in the developing world. Zidovudine is also relatively available and has many therapeutic advantages but also has a risk of producing mitochondrial toxicity. As a result, it will often be necessary to add drugs to manage cardiovascular risk and treat the metabolic side effects of the HAART regimen and HIV infection.

\section{Statin Therapy}

We often think of statins, through inhibition of HMG-CoA reductase, primarily affecting cholesterol synthesis, a fairly distal effect in the disturbances of adipocyte function described above. From that theoretical standpoint, they would not be an ideal treatment modality for HIV dyslipidemia, as they do not directly counteract the effects on visceral adipocyte maturation, adiponectin or leptin dysregulation, or cortisol levels and effects, and do not directly affect angiotensin synthesis in adipocytes with resultant hypertension. However, statin pluripotential effects include anti-inflammatory activity and downregulation of CRP production, thus decreasing macrophage migration, so it may indirectly affect a number of lipodystrophy effects. In fact, statins have been shown in some studies to affect fat maldistribution patterns positively and have a positive effect on lipoatrophy. Because CRP likely plays a key role in several pathways of HIV lipodystrophy and dyslipidemia, as well as contributing to atherosclerotic progression, it makes sense that statins would be effective therapy in more ways than just lowering LDL and triglycerides and modestly raising HDL cholesterol. In one experimental model, they seem also to inhibit HIV infection [18], thus theoretically having the potential to serve as a synergistic agent in ART, although unproven by clinical data at this point. As a class, they are effective in terms of affecting cardiovascular risk parameters, with reasonably low side effect profile. As in the general population, statins do seem to be the first-line agents for 
the treatment of dyslipidemias, especially for patients with predominance of high LDL cholesterol levels, but also for those presenting with moderate triglyceride elevations and low HDL cholesterol. There are significant drug interaction issues with all statins with patients on ART. Because all protease inhibitors affect CYP3a4 levels, in particular lopinavir/ritonavir, it generally is a good idea in patients on HAART regimens that include protease inhibitors, to choose statins that are less heavily metabolized by this system. The goal is to avoid the potential for increased statin toxicity due to excessive levels that might result from drug metabolism interference from the antiviral regimen. Although lovastatin, simvastatin, and (to a lesser extent) atorvastatin are heavily metabolized by CYP3a4 [19], pravastatin, rosuvastatin, and fluvastatin are less heavily dependent on CYP3a4 and should have less risk of toxicity when combined with protease inhibitors. Pravastatin has relatively less potency, but evidence supports rosuvastatin in particular as being clinically effective in lowering LDL cholesterol and triglycerides and raising HDL cholesterol. Despite the fact that rosuvastatin is less heavily metabolized by CYP3a4, levels have been reported up to twice normal on patients taking protease inhibitors, so the dose should be started low $(10 \mathrm{mg}$ ) and only raised to $20 \mathrm{mg}$ cautiously [20]. Another possible strategy is to change the protease inhibitor, if possible, to atazanavir, which is less likely to boost rosuvastatin levels. Fluvastatin, although less potent in lower-dose range, has the least incidence of myopathy and hepatic toxicity, and also is less dependent on CYP3a4 metabolism. There is less evidence for fluvastatin in HIV patients on HAART, but it may also be a good candidate. In patients on NNRTIs, especially efavirenz, the statin levels may need to be increased as CYP3a4 levels are boosted by the NNRTIs. For patients who have refractory dyslipidemias, this has some evidence as a strategy to increase the potency of the statin therapy.

\section{Other Therapeutic Options}

For patients with hypertriglyceridemia ( $>500 \mathrm{mg} / \mathrm{dL}$ ), fibrates may be the first-line choice. Fortunately, their metabolism does not depend on CYP3a4 and there is less of an issue of drug interaction. Co-treatment with fibrates and statins does carry a risk or hepatotoxicity, however.

Treatment with fish oils, other sources of omega 3 fatty acids, or niacin does not seem to be particularly affected by HIV infection or ART, and they remain viable treatment options, especially in patients with high triglycerides and low HDL as their lipid problems. For patients taking medications that affect bleeding time, or with immune thrombocytopenia, fish oils also increase bleeding time and should be used cautiously or avoided [21]. Binding resins may adversely affect antiretroviral drug absorption and thus should be avoided in patients receiving ART. There are some indications ezetimibe may aid in preventing absorption of cholesterol from the gut without affecting drug absorption, and it should be considered as an agent to improve cholesterol control [22].

Other potential targets for treatment of HIV-related metabolic disorders include insulin resistance and adipocyte dysmaturity. Insulin resistance may contribute to lipoatrophy, and has been treated in the non-HIV population suffering from metabolic syndrome with the PPAR agonist thiazolidinediones and with metformin. Although there is evidence of efficacy for the thiazolidinedione drug rosiglitazone in decreasing insulin resistance associated with modest improvements in lipoatrophy [23], there are concerning effects reported for rosiglitazone of increasing cholesterol and worsening risk of myocardial infarction. This would make it a less favorable drug in a population already at risk [24]. Several trials of rosiglitazone, though, failed to control for concomitant intake of thymidine analog and NRTIs, which may have nullified the beneficial effect of PPAR stimulation. Thiazolidinediones in general, however, may be less effective in HIV patients on ART than the general population with metabolic syndrome [25]. (Discussion of rosiglitazone may be a moot point since it is in the process of being pulled from the market as this manuscript is being written.) Pioglitazone does not seem to carry the risks of increasing LDL and would seem to be a more favorable member of the thiazolidinedione class for consideration [26]. Metformin may be an even more effective intervention for both lipodystrophic change and dyslipidemia. In a recent meta-analysis covering 16 separate trials comparing rosiglitazone, pioglitazone and metformin, there was much more robust evidence for benefits of metformin over insulin and triglyceride levels and some degree of reversion to some of the lipodystrophic changes. Other experimental strategies include administration of leptin, especially in patients with demonstrated hypoleptinemia and the use of high physiologic level growth hormone administration. Both of these showed some promise with HIV lipodystrophy, with improvements in visceral body fat, insulin resistance, and serum lipid profiles. No severe or frequent side effects were observed, and there was no net increase in glucose, $\mathrm{HbAlc}$, or glucose intolerance. At this point, approval as therapy and larger-scale trials have not been done, but these do hold promise for potential treatment modalities for HIV-associated lipid syndromes [2732].

\section{Endothelial Dysfunction, HIV Infection, and Therapy}

Studies of endothelial dysfunction in treatment-naïve HIVpositive patients showed improvement after initiation of 
ART [33]. However, studies of patients receiving protease inhibitors also show worsening of parameters of endothelial function with longer therapy exposures [34]. Although an assumption can be made that endothelial dysfunction observed in treatment-naïve patients with HIV is mediated by an inflammatory mechanism, direct viral damage to the endothelium, or both [35], the exact sequence or mechanism of this inflammation has not been full elucidated. A role for infectious agents might also be suspected.

\section{Suggestions for Clinical Practice}

As part of the practice of initiating HAART, getting a thorough history of cardiovascular risk factors, family history, and personal history of dyslipidemias should be a part of initial ART choice. Although the evidence is conflicting, patients with significant risk factors might fare better on protease-sparing regimens, even as initial therapy. Predictors of dyslipidemia in HIV patients include a family history positive for dyslipidemias, sedentary lifestyle, and abnormal body mass index. Unfortunately, presence or absence of such a history has limited predictive value for the development of ART-associated dyslipidemias or lipodystrophy.

It is also important for the treating physician to look for co-infection with hepatitis C. Co-infection with hepatitis $\mathrm{C}$ is relatively sparing of hypercholesterolemia or lipodystrophy but also poses potential problems of increased vulnerability to hepatic damage from hepatotoxic drugs. Hepatitis C co-infection has serious implications for management of HIV-positive patients and choices of antiviral priorities.

In patients who have dyslipidemia, with or without associated fat maldistribution, one should ask the question if initial therapy should be with alteration of the HAART regimen or addition of antihyperlipidemic agents, such as statins. In many cases, the first priority is an effective antiviral regimen. If one can choose from effective regimens, one that is least likely to exacerbate lipodystrophy effects would be a beneficial first step. If the patient already has risk factors for atherosclerosis and/or manifestations of dyslipidemia and lipodystrophy, including a statin chosen for minimal risk of drug-drug interactions would be a next best step.

HIV patients, especially those on HAART, are more susceptible to drug-drug interactions and adverse drug reactions than the general population, and it is wise to monitor for adverse events with statins and other drugs that may ensue due to an increase in drug levels caused by interference in drug metabolism. Even rosuvastatin, which theoretically is not metabolized by CYP3A4, has shown increased levels with patients receiving protease inhibitors as part of HAART. Myositis, especially liver enzyme abnormalities, is not unusual in HIV patients on multiple medications and always requires thoughtful evaluation.

\section{Conclusions}

HIV infection is now a chronic manageable disease, and cardiovascular risk is definitely one of the things that need to be managed for quality of life and longevity. Lipodystrophy and dyslipidemia are risk factors that can be addressed, both for the quality of life of the patient and in prevention of cardiovascular disease. It should not be neglected among the flurry of considerations that need to be followed for these patients.

Disclosure J. Gregory Troll reports no potential conflict of interest relevant to this article.

Open Access This article is distributed under the terms of the Creative Commons Attribution Noncommercial License which permits any noncommercial use, distribution, and reproduction in any medium, provided the original author(s) and source are credited.

\section{References}

Papers of particular interest, published recently, have been highlighted as:

- Of importance,

•. Of major importance

1. Seigneur, M, Constans, J, Blann, A, et al. Soluble adhesion molecules and endothelial cell damage in HIV infected patients. Thromb Haemost 1997; 77:646.

2. Yu-Min P. Shen, MD, Eugene P. Frenkel, MD Thrombosis and a Hypercoagulable State in HIV-Infected Patients; doi: 10.1177/ 107602960401000311 CLIN APPL THROMB HEMOST July 2004 vol. 10 no. 3 277-280

3. Karmochkine, M, Ankri, A, Calvez, V, et al. Plasma hypercoagulability is correlated to plasma HIV load. Thromb Haemost 1998; 80:208

4. - Grunfeld, C, Delaney, JA, Wanke, C, et al. Preclinical atherosclerosis due to HIV infection: carotid intima-medial thickness measurements from the FRAM study. AIDS 2009; 23:1841. This article provides evidence for the effect of HIV infection, rather than HAART, as a causative agent in atherogenesis.

5. - Kaplan, RC, Kingsley, LA, Gange, SJ, et al. Low CD4+ T-cell count as a major atherosclerosis risk factor in HIV-infected women and men. AIDS 2008; 22:1615. This article provides more evidence of the importance of HIV infection as part of the process of atherogenesis. The correlation with CD4+ T cell count suggests either viremia (though not measured directly) or perhaps immunodeficiency as a factor. Immunodeficiency could act as an atherogenic agent either due to inflammatory process or unidentified, uncontrolled vasculopathic infection. 
6. Triant, VA, Meigs, JB, Grinspoon, SK. Association of C-Reactive Protein and HIV Infection With Acute Myocardial Infarction. J Acquir Immune Defic Syndr 2009; 51:268.

7. F. De Lorenzo, S. Collot-Teixeira, et al. Metabolic-Inflammatory Changes, and Accelerated Atherosclerosis in HIV Patients: Rationale for Preventative Measures Current Medicinal Chemistry, 2008, 15, 2991-2999 2991 0929-8673/08 (C) 2008 Bentham Scie nce Publishers Ltd.

8. Francisci, D, Giannini, S, Baldelli, F, et al. HIV type 1 infection, and not short-term HAART,induces endothelial dysfunction. AIDS 2009; 23:589

9. •- Grinspoon, SK, Grunfeld, C, Kotler, DP, et al. State of the science conference: Initiative to decrease cardiovascular risk and increase quality of care for patients living with HIV/AIDS: executive summary. Circulation 2008; 118:198). This is an excellent discussion of relevant articles reviewing the state of the science on this subject.

10. Mondy, K.; Overton, E.T.; Grubb, J, et al. Metabolic syndrome in HIV-infected patients from an urban, midwestern US outpatient population. Clinical Infections Dis., 2007, 44, 726-34.

11. Pullinger CR, Aouizerat BE, Gay C, et al. Metabolic abnormalities and coronary heart disease risk in human immunodeficiency virusinfected adults. Metab Syndr Relat Disord. 2010 Jun; 8(3):279-86.

12. Joly, V, Flandre, $P$, Meiffredy, V, et al. Increased risk of lipoatrophy under stavudine in HIV-1-infected patients: results of a substudy from a comparative trial. AIDS 2002; 16:2447

13. Moyle, GJ, Sabin, CA, Cartledge, J, et al. A randomized comparative trial of tenofovir DF or abacavir as replacement for a thymidine analogue in persons with lipoatrophy. AIDS 2006; 20:2043.

14. El Hadri K, Glorian M, Monsempes C, Dieudonné MN, et al. In vitro suppression of the lipogenic pathway by the nonnucleoside reverse transcriptase inhibitor efavirenz in $3 \mathrm{~T} 3$ and human preadipocytes or adipocytes. Chem. 2004 Apr 9;279(15):1513041. Epub 2004 Jan 13.

15. Biol Res Nurs. 2007 Jan;8(3):177-85. Effects of high-intensity endurance and resistance exercise on HIV metabolic abnormalities: a pilot study. Robinson FP, Quinn LT, Rimmer JH.

16. Martin, A, Smith, DE, Carr, A, et al. Reversibility of lipoatrophy in HIV-infected patients 2 years after switching from a thymidine analogue to abacavir: the MITOX Extension Study. AIDS 2004; $18: 1029$.

17. McComsey, GA, Ward, DJ, Hessenthaler, SM, et al. Improvement in lipoatrophy associated with highly active antiretroviral therapy in human immunodeficiency virus-infected patients switched from stavudine to abacavir or zidovudine: the results of the TARHEEL study. Clin Infect Dis 2004; 38:263

18. Amet T, Nonaka M, Dewan MZ, et al. Statin-induced inhibition of HIV-1 release from latently infected U1 cells reveals a critical role for protein prenylation in HIV-1 replication. Microbes Infect. 2008 Apr;10(5):471-80. Epub 2008 Jan 20.

19. Dube, MP, Stein, JH, Aberg, JA, et al. Guidelines for the evaluation and management of dyslipidemia in human immunodeficiency virus (HIV)-infected adults receiving antiretroviral therapy: recommendations of the HIV Medical Association of the Infectious Disease Society of America and the Adult AIDS Clinical Trials Group. Clin Infect Dis 2003; 37:613.
20. Brewer, HB Jr. Benefit-risk assessment of Rosuvastatin 10 to 40 milligrams. Am J Cardiol 2003; 92:23K

21. Gerber, JG, Kitch, DW, Fichtenbaum, CJ, et al. Fish Oil and Fenofibrate for the Treatment of Hypertriglyceridemia in HIVInfected Subjects on Antiretroviral Therapy: Results of ACTG A5186. J Acquir Immune Defic Syndr 2008; 47:459

22. Wohl, DA, Waters, D, Simpson, RJ Jr, et al. Ezetimibe alone reduces low-density lipoprotein cholesterol in HIV-infected patients receiving combination antiretroviral therapy. Clin Infect Dis $2008 ; 47: 1105$.

23. Macallan DC, Baldwin C, Mandalia S, et al. Treatment of altered body composition in HIV-associated lipodystrophy: comparison of rosiglitazone, pravastatin, and recombinant human growth hormone. HIV Clin Trials. 2008 Jul-Aug; 9(4):254-68.

24. Nissen, SE, Wolski, K. Effect of rosiglitazone on the risk of myocardial infarction and death from cardiovascular causes. N Engl J Med 2007; 356:2457.

25. Grinspoon, S. Use of Thiazolidinediones in HIV-Infected Patients: What Have We Learned? J Infect Dis 2007; 195:1731

26. Mallon, PW, Sedwell, R, Rogers, G, et al. Effect of rosiglitazone on peroxisome proliferatoractivated receptor gamma gene expression in human adipose tissue is limited by antiretroviral drug-induced mitochondrial dysfunction. J Infect Dis 2008; 198:1794.

27. Slama, L, Lanoy, E, Valantin, MA, et al. Effect of pioglitazone on HIV-1-related lipodystrophy: a randomized double-blind placebocontrolled trial (ANRS 113). Antivir Ther 2008; 13:67

28. Sheth SH, Larson RJ. The efficacy and safety of insulin-sensitizing drugs in HIV-associated lipodystrophy syndrome: a meta-analysis of randomized trials. BMC Infect Dis. 2010 Jun 23;10:183.

29. Hansen BR, Haugaard SB, Jensen FK, et al. Long-term highphysiological-dose growth hormone reduces intra-abdominal fat in HIV-infected patients with a neutral effect on glucose metabolism. HIV Med. 2010 Apr;11(4):266-75. Epub 2009 Dec 8.

30. Falutz J, Potvin D, Mamputu JC, et al. Effects of tesamorelin, a growth hormone-releasing factor, in HIV-infected patients with abdominal fat accumulation: a randomized placebo-controlled trial with a safety extension. J Acquir Immune Defic Syndr. 2010 Mar 1;53(3):311- 22

31. Wang Y, Tomlinson B. Expert Tesamorelin, a human growth hormone releasing factor analogue. Opin Investig Drugs. 2009 Mar;18(3):303-10.

32. Falutz J, Allas S, Mamputu JC, et al. Long-term safety and effects of tesamorelin, a growth hormone-releasing factor analogue, in HIV patients with abdominal fat accumulation. AIDS. 2008 Sep 12;22(14):1719-28

33. Lafeuillade A, Alessi MC, Poizot-Martin I, et al. Endothelial cell dysfunction in HIV infection. J Acquir Immune Defic Syndr. 1992;5(2):127-31.

34. Ferraro S, Paolillo S, Gargiulo M, et al. Effect of antiretroviral therapy on carotid intima-media thickness in HIV-infected patients. G Ital Cardiol (Rome). 2009 Sep;10(9):596-601.

35. J Acquir Immune Defic Syndr. 2009 September 1; 52(1): 25-31. Untreated HIV Infection and Large and Small Artery ElasticityJason V. Baker, MD, MS, ${ }^{*} \dagger$ Daniel Duprez, MD, PhD, $†$ Joshua Rapkin, MS, $\ddagger$ Katherine Huppler Hullsiek, PhD, $\$$ Harrison Quick, $\mathrm{BS}, \ddagger$ Richard Grimm, MD, PhD, James D. Neaton, PhD, and Keith Henry, MD 